\title{
Article
}

\section{Loneliness and the recognition of vocal socioemotional expressions in adolescence}

Morningstar, Michele, Nowland, Rebecca, Dirks, Melanie A. and Qualter, Pamela

Available at http://clok.uclan.ac.uk/30551/

Morningstar, Michele, Nowland, Rebecca ORCID: 0000-0003-4326-2425, Dirks, Melanie A. and Qualter, Pamela (2020) Loneliness and the recognition of vocal socioemotional expressions in adolescence. Cognition and Emotion, 34 (5). pp. 970-976. ISSN 0269-9931

It is advisable to refer to the publisher's version if you intend to cite from the work. http://dx.doi.org/10.1080/02699931.2019.1682971

For more information about UCLan's research in this area go to http://www.uclan.ac.uk/researchgroups/ and search for <name of research Group>.

For information about Research generally at UCLan please go to http://www.uclan.ac.uk/research/

All outputs in CLoK are protected by Intellectual Property Rights law, including Copyright law. Copyright, IPR and Moral Rights for the works on this site are retained by the individual authors and/or other copyright owners. Terms and conditions for use of this material are defined in the policies page.

\section{CLoK}

Central Lancashire online Knowledge www.clok.uclan.ac.uk

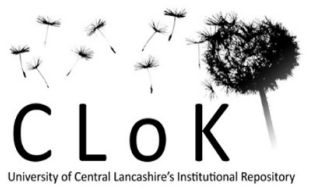


Loneliness and the Recognition of Vocal Socioemotional Expressions in Adolescence

\author{
Author Accepted Manuscript \\ Michele Morningstar ${ }^{1,4}$, Rebecca Nowland ${ }^{2}$, Melanie A. Dirks ${ }^{1}, \&$ Pamela $_{\text {Qualter }}^{3}$
}

1. Department of Psychology, McGill University, Canada

2. School of Nursing, University of Central Lancashire, UK

3. Manchester Institute of Education, University of Manchester, UK

4. Research Institute at Nationwide Children's Hospital, The Ohio State University, USA

This is the author accepted manuscript. It is advisable to refer to the publisher's version of the paper if you intend to cite from the work. The article has been accepted for publication in Cognition and Emotion.

Running title: LONELINESS \& VOCAL EMOTION RECOGNITION

Correspondence concerning this article should be addressed to:

Michele Morningstar, Ph.D.

Address: 700 Children's Drive, Nationwide Children's Hospital, Columbus OH 43201

Email: michele.morningstar@ nationwidechildrens.org

Phone: 614-722-4727

ORCiD: 0000-0002-5715-1446 


\title{
LONELINESS \& VOCAL EMOTION RECOGNITION
}

\begin{abstract}
Lonely individuals show increased social monitoring and heightened recognition of negative facial expressions. The current study investigated whether this pattern extends to other nonverbal modalities by examining associations between loneliness and the recognition of vocal emotional expressions. Youth, ages 11 to 18 years $(n=122)$, were asked to identify the intended emotion in auditory portrayals of basic emotions (anger, disgust, fear, happiness, sadness) and social expressions (friendliness, meanness). Controlling for social anxiety, age, and gender, links between loneliness and recognition accuracy were emotion-specific: loneliness was associated with poorer recognition of fear, but better recognition of friendliness. Lonely individuals' motivation to avoid threat may interfere with the recognition of fear, but their attunement to affiliative cues may promote the identification of friendliness in affective prosody. Monitoring for social affiliation cues in others' voices might represent an adaptive function of the reconnection system in lonely youth, and be a worthy target for intervention.
\end{abstract}

Key words: emotion recognition; affective prosody; vocal communication; social monitoring; social information processing 


\section{LONELINESS \& VOCAL EMOTION RECOGNITION}

Loneliness is associated with heightened social monitoring (Gardner, Pickett, Jefferis, \& Knowles, 2005; Spithoven, Bijttebier, \& Goossens, 2017). This attention bias means that individuals prioritize social information (Gardner et al., 2005), showing sensitivity to both (1) negative stimuli that signal social threat and (2) positive cues that represent opportunities for reconnection (Qualter et al., 2015). Lonely adolescents and adults have been shown to better recall positive and negative social events over non-social information (Gardner et al., 2005), and to better recognize negative emotions in facial expressions (e.g., anger, fear, or sadness; Lodder, Scholte, Goossens, Engels, \& Verhagen, 2016; Vanhalst, Gibb, \& Prinstein, 2017). However, less is known about the association between loneliness and the recognition of socio-emotional expressions in other nonverbal modalities, such as vocal prosody. Decoding emotional intent in others' voices follows a different developmental trajectory than the recognition of facial expressions, and likely engages different cognitive processes (Morningstar, Nelson, \& Dirks, 2018). To extend understanding of how loneliness is associated with social information processing, the current study investigated links between feelings of loneliness and the recognition of vocal affect in adolescents.

Loneliness is characterized by negative affect experienced when there is a discrepancy between desired and existing social relationships (Perlman \& Peplau, 1981). According to current theories (Cacioppo \& Hawkley, 2009; Qualter et al., 2015; Spithoven et al., 2017), loneliness motivates individuals to reconnect, but — paradoxically — is also characterized by hypervigilance to social threat, and by social withdrawal. To reconnect with others and overcome negative affect, lonely individuals are initially inclined to withdraw socially, providing time and space to evaluate their current social situations. Loneliness also activates specific cognitive processes that help generate behavioural responses that avoid further social harm and increase 


\section{LONELINESS \& VOCAL EMOTION RECOGNITION}

social reconnection and inclusion. For example, social information is prioritized over non-social information (Gardner et al., 2005), helping the lonely person react faster to information in the social environment and make rapid decisions about the intent of potential social partners (Spithoven et al., 2017). These attentional biases are thought to be engaged very quickly, although the time-course of attention to social stimuli in lonely people may vary by developmental stage: for instance, lonely young adults showed initial vigilance to social threat cues, but lonely children demonstrated both hypersensitivity to, and difficulty disengaging their attention from, those cues (Qualter et al., 2015).

To understand the consequences of lonely people's attention biases on other aspects of social information processing, a number of studies have examined whether loneliness is linked to the ability to recognize emotions - in morphed faces that increase in emotional intensity (Lodder et al., 2016; Vanhalst et al., 2017), in low- and high-intensity emotional faces (Gardner et al., 2005; Knowles, Lucas, Baumeister, \& Gardner, 2015), or in short movies and images of emotional faces (Zysberg, 2012). Findings are mixed: loneliness has been associated with increased ER accuracy (Gardner et al., 2005), decreased ER accuracy (Zysberg, 2012), or decreased ER accuracy only under certain conditions (i.e., social framing; Knowles et al., 2015). However, the use of global ER scores (collapsing across negative and positive faces) obscures potential emotion-specific associations between loneliness and ER (Spithoven et al., 2017). Only two studies have examined accuracy for different emotions separately: Lodder and colleagues (2016) found that loneliness was associated with increased recognition of angry faces (but not of fearful, sad, or happy faces), whereas Vanhalst and colleagues (2017) found that lonely adolescents were better able to detect sadness and fear (as well as happiness to some extent, but 


\section{LONELINESS \& VOCAL EMOTION RECOGNITION}

not anger) in faces. These findings suggest that lonely individuals may show heightened capacity to identify negative facial expressions, though emotion-specific effects are not consistent.

To date, research examining the relationship between loneliness and ER has almost exclusively assessed the interpretation of facial expressions. However, a speaker's tone of voice also contains important information about their emotional state or social attitudes (Johnstone \& Scherer, 2000). Decoding emotional information in others' tone of voice likely engages different cognitive processes than the recognition of facial emotion (Morningstar, Nelson, et al., 2018). For instance, whereas sufficient information for emotion recognition is present in faces at any one point in time, vocal prosody requires the integration of rapidly changing information across a longer time frame.

To our knowledge, only one study has examined the association between loneliness and vocal ER (Knowles et al., 2015). Adult listeners heard words being spoken in a negative or positive tone of voice, and were asked to categorize the valence of the word's intonation (rather than its content). Participants were either told that this task measured a social skill that was important for social success (social framing condition), or a cognitive skill that was important for academic success (nonsocial condition). Loneliness was associated with poorer accuracy only in the social framing condition, suggesting that vocal ER was disrupted when anxiety about social performance was manipulated. However, the relationship between loneliness and the recognition of positive and negative emotions was not examined separately.

In addition, no study has investigated how vocal ER skills are related to loneliness in adolescence. The ability to recognize vocal affect follows a more protracted developmental trajectory than does facial ER (Chronaki, Hadwin, Garner, Maurage, \& Sonuga-Barke, 2015; Morningstar, Nelson, et al., 2018), continuing to improve through mid-adolescence 


\section{LONELINESS \& VOCAL EMOTION RECOGNITION}

(Morningstar, Ly, Feldman, \& Dirks, 2018). Moreover, the percentage of individuals reporting feelings of loneliness increases around puberty, affecting over $26 \%$ of adolescents (Rönkä, Rautio, Koiranen, Sunnari, \& Taanila, 2014). Adolescence is a period of increased engagement with peers and heightened sensitivity to cues of social rejection or affiliation (Nelson et al., 2005). Youth who struggle to interpret emotional intent in complex nonverbal cues, such as others' tone of voice, may find it particularly challenging to connect with others socially. Determining how lonely youth respond to emotional prosody is important to assess the generalizability of current models of loneliness and provide a complete understanding of this group's social information processing, with implications for intervention.

\section{Goals \& hypotheses}

The current study examines emotion-specific associations between loneliness and vocal recognition accuracy from early- to late-adolescence, a developmental period in which changes in social behaviour, feelings of loneliness, and vocal ER skills are expected to occur. We asked 11- to 18-year-old youth to indicate the expression conveyed in auditory portrayals of five basic emotions (happiness, sadness, fear, anger, and disgust) and the social expressions of 'meanness' and 'friendliness'. The latter two expressions can be conceptualized as representing cues of rejection and affiliation, respectively, and have been shown to differ acoustically (Morningstar, Dirks, \& Huang, 2017) and perceptually (Morningstar, Ly, et al., 2018) from other basic emotions. Including such expressions taps into listeners' understanding of socially relevant nonverbal cues, which are likely pertinent and commonly expressed within close relationships, beyond prototypical "basic" emotions.

Given the sparse nature of previous work on loneliness and vocal emotion recognition, our exploratory hypotheses were based primarily on theory and findings pertaining to facial ER. 


\section{LONELINESS \& VOCAL EMOTION RECOGNITION}

Prior findings suggest a potential main effect of loneliness on recognition accuracy (Gardner et al., 2005; Knowles et al., 2015; Zysberg, 2012); however, given reports of - and calls for tests of-emotion-specific associations between loneliness and ER (Lodder et al., 2016; Vanhalst et al., 2017; Spithoven et al., 2017), we also tested for an interaction between loneliness and emotion type on accuracy. Because social anxiety is associated with vocal ER in children (McClure \& Nowicki, 2001) and moderates the link between loneliness and vocal ER in adults (Knowles et al., 2015), we controlled for social anxiety in our analyses. In addition, we controlled for age and gender due to known associations between those variables and both loneliness-related social information processing biases and emotion recognition skills (Herba \& Phillips, 2004; Qualter et al., 2015).

\section{Method}

\section{Participants}

Participants were youth attending a Summer School at a university in England, where they completed several experimental studies. Parental consent for Summer School attendance was provided. A total of 122 youth (57.38\% female; $94.57 \%$ of attendees), ages $11-18$ years $(M$ $=15.39$ years old, $S D=1.77$ ) assented or consented to take part in the current study. Institutional Research Ethics Boards approved all procedures.

\section{Measures}

Loneliness. Youth completed the Loneliness and Aloneness Scale for Children and Adolescents questionnaire (Marcoen, Goossens, \& Caes, 1987). This 48-item questionnaire comprises 4 subscales: two assessing loneliness (parent loneliness, peer loneliness), and two assessing aloneness (aversion to aloneness, affinity for aloneness). The sum of scores for the 


\section{LONELINESS \& VOCAL EMOTION RECOGNITION}

parent $(\alpha=0.87)$ and peer loneliness $(\alpha=0.89)$ subscales was used in analyses $(M=103.41, S D$ $=17.75)$.

Social anxiety. Youth completed the 22-item Social Anxiety Measures for Children and Adolescents (La Greca \& Stone, 1993). The total score for social anxiety symptomatology $(\alpha=$ $0.95)$ was included as a covariate $(M=48.01, S D=16.45)$.

Vocal emotion recognition. The vocal ER task comprised audio recordings produced by actors in a previous study (AUTHOR BLINDED). Actors spoke standardized-content sentences (e.g., "I didn't know about it", "Why did you do that?”) in different emotional tones of voice. Participants heard 140 recordings of socio-emotional expressions (anger, disgust, fear, friendliness, happiness, meanness, and sadness) produced by adolescent and adult actors (7 expressions x 5 sentences x 2 speaker ages, spoken by both male and female actors), and selected the intended expression from 7 labels. Recordings were selected from the full set of available stimuli (AUTHOR BLINDED) based on judges' ratings of their recognizability and authenticity (AUTHOR BLINDED). Previous work with this task has shown emotion-specific recognition patterns consistent with prior findings (e.g., anger and sadness are best recognized, and happiness and disgust are more poorly recognized; Johnstone \& Scherer, 2000), as well as expected age-related increases in accuracy (AUTHOR BLINDED).

All 140 recordings were presented to listeners through headphones, in a randomized order, using E-Prime. Participants heard each recording twice in a row. They were then asked to select the speaker's intended expression by pressing labelled keys on a keyboard. Participants' accuracy in identifying each emotion was calculated using the unbiased hit rate $\left(\mathrm{H}_{\mathrm{u}}\right.$; Wagner, 1993), which indexes accuracy correcting for response bias. A value of 1 for $\mathrm{H}_{\mathrm{u}}$ indicates perfect 


\section{LONELINESS \& VOCAL EMOTION RECOGNITION}

recognition (100\% hit rate, without false alarms or false negatives), whereas a value of 0 indicates no recognition of an expression ( $0 \%$ hit rate, with only false alarms and false negatives). $\mathrm{H}_{\mathrm{u}}$ values were arcsine transformed before use in analyses (Wagner, 1993; $M=0.96$, $S D=0.18)^{1}$. The resulting variables were screened for skewness and kurtosis, and distributions were sufficiently normal.

\section{Analysis}

A general linear model (GLM) was performed in SPSS v.24 to examine the effects of Emotion (within-subjects: anger, disgust, fear, friendliness, happiness, meanness, sadness), Loneliness (between-subjects: mean-centered, continuous), Gender (between-subjects: male, female), Age (between-subjects: mean-centered, continuous), and Social Anxiety scores (between-subjects: mean-centered, continuous) on listeners' accuracy $\left(\mathrm{H}_{\mathrm{u}}\right)^{2}$. All variables were entered simultaneously into the model. Greenhouse-Geisser corrections were applied as indicated by Mauchly's test of sphericity $(p<.05)$. We controlled for family-wise error rate by applying Holm-Bonferroni corrections (Holm, 1979) to the GLM. Power calculations suggested that the sample size afforded over $80 \%$ power to detect small effects.

\section{Results}

Table 1 contains results of the full factorial model. There were no main effects of Loneliness, Society Anxiety, or Age, but there was a main effect of Emotion. Post-hoc pairwise comparisons with Šidák corrections revealed that anger was the best recognized expression $(M=$

\footnotetext{
${ }^{1}$ Raw accuracy estimates for each emotion are provided in Supplemental Materials, Table 1.

${ }^{2}$ To verify the robustness of our findings, additional models were performed a) without social anxiety as a covariate, b) including the age of the speaker as a predictor, and c) using uncorrected estimates of accuracy instead of $\mathrm{H}_{\mathrm{u}}$ as the measure of performance. Results are identical to those presented in text (see Supplemental Materials, Tables 2-4), with the following exceptions: loneliness is not associated with the recognition of fear when social anxiety is removed as a covariate, and age is not associated with the recognition of fear when uncorrected estimates of accuracy are used instead of $\mathrm{H}_{\mathrm{u}}$.
} 


\section{LONELINESS \& VOCAL EMOTION RECOGNITION}

$1.38, S E=0.02)$, followed by sadness $(M=1.18, S D=0.03)$, fear $(M=0.96, S D=0.03)$, friendliness $(M=0.93, S D=0.02)$, happiness $(M=0.88, S D=0.03)$, meanness $(M=0.74, S D=$ $0.03)$, and disgust $(M=0.68, S D=0.02)$. Fear, friendliness, and happiness did not differ from one another $(p s>.05)$, nor did disgust and meanness $(p=.39)$. All other expressions were significantly different from all others, $p s<.05$. A main effect of Gender, such that female listeners were more accurate than males, did not survive Holm-Bonferroni corrections.

There was a significant interaction between Emotion and Loneliness. Parameter estimates suggested that greater loneliness was associated with poorer recognition of fear, $\beta=-.24, t(116)$ $=-2.09, p=.04,95 \% \mathrm{CI}[-.46,-.01]$, and heightened recognition of friendliness, $\beta=.29, t(116)=$ $2.51, p=.01,95 \%$ CI $[.06, .53]$ (Figure 1). Emotion also interacted with Age, with parameter estimates suggesting that age was positively associated with the recognition of sadness, $\beta=.32$, $t(116)=3.50, p=.001,95 \%$ CI $[.14, .50]$, and fear, $\beta=.18, t(116)=2.00, p=.048,95 \%$ CI $[<.01, .37]$. An interaction between Emotion and Social Anxiety did not survive corrections.

\section{Discussion}

The current study was the first to examine whether loneliness was associated with vocal emotion recognition (ER) skills in adolescents. Results suggest that links between loneliness and vocal ER are emotion-specific. Loneliness was associated with poorer recognition of fear, but with better recognition of friendliness, in others' voices. The results of this study highlight the importance of comparing lonely youth's ability to recognize different emotions separately, and in both facial and vocal modalities, to better understand the impact of loneliness on social information processing.

Consistent with previous work on vocal ER (Johnstone \& Scherer, 2000; Morningstar, Ly, et al., 2018), we found that anger and sadness were most accurately identified, whereas 


\section{LONELINESS \& VOCAL EMOTION RECOGNITION}

disgust, happiness, and meanness were poorly recognized. Age was positively associated with accuracy for sadness and fear. This finding is concordant with prior evidence that the recognition of those emotions emerges later in development (Chronaki et al., 2015; Herba \& Phillips, 2004). In contrast, the recognition of other vocal emotions may plateau in adolescence, though more research is needed on the developmental trajectory of non-facial ER skills in youth.

Building on previous literature, we found emotion-specific associations between loneliness and vocal ER. Contrary to previous work with emotional faces (Lodder et al., 2016; Vanhalst et al., 2017), loneliness was linked to poorer recognition of vocal fear, which-like anger — can be conceptualized as socially threatening (Green \& Phillips, 2004). A negative relationship between loneliness and vocal ER is broadly consistent with prior work showing that lonely individuals performed worse on a vocal recognition task under certain conditions (Knowles et al., 2005). However, loneliness was also associated with more accurate identification of friendliness. This finding supports current theories suggesting that loneliness increases social monitoring to promote sensitivity to potential opportunities for social reconnection (Gardner et al., 2005; Qualter et al., 2015), such as a friendly tone of voice. Associations with other positive or socially threatening voices, such as happiness $(\beta=.21, p=$ $.08)$ or anger $(\beta=-.18, p=.13)$, were not significant but were consistent with the above findings. It is possible that the inclusion of social expressions like friendliness and meanness may have impacted listeners' ability to identify those conceptually similar emotions.

Increased recognition of social threat (or negative emotions more broadly) may only be evident among lonely people when faces are used as stimuli rather than voices, due to temporal differences in the processing of both types of stimuli. Attention to threat in other populations, such as anxious youth, has been found to be modulated by the time-course of stimulus 


\section{LONELINESS \& VOCAL EMOTION RECOGNITION}

presentation (Yiend, 2010)—such that longer stimuli provoke initial attention to, and subsequent avoidance of, threatening cues. A similar process may be at play with vocal stimuli that inherently unfold over time. Lonely youth may initially tune into, then avoid, threatening stimuli (e.g., fearful voices) — which may interfere with correct recognition of the intended emotion. With positive prosodic modulations, such as those of a friendly voice, that same process may engage listeners' attention longer without triggering avoidance, and thus enhance recognition accuracy. Prolonged engagement with social cues may thus be an important mechanism through which the reconnection mechanism is deployed in lonely youth. Testing that question in the auditory modality by comparing responses to emotional sentences versus short vocal bursts, in both dot-probe and explicit emotion recognition tasks, could help characterize the interplay between social monitoring, attention biases, and ER in lonely youth.

\section{Strengths, limitations, and future directions}

The current study extends our understanding of the association between social information processing and loneliness by (1) examining the relationship between those variables in adolescence, (2) highlighting potential modality- and emotion-specific effects, and (3) extending our understanding of reaffiliation processes related to loneliness (Qualter et al., 2015). Testing models of loneliness and social information processing in a developmental context is important, given strong evidence of age-related effects on both constructs and their interaction. We further built on existing work in adults (Knowles et al., 2015) by examining emotion-specific associations between loneliness and the recognition of a broad range of positive and negative expressions in the voice - including cues of social affiliation and rejection, to which lonely individuals have been found to be particularly sensitive (Qualter et al., 2013). Our results are consistent with current theoretical propositions that lonely people are attuned to social 


\section{LONELINESS \& VOCAL EMOTION RECOGNITION}

threat/negative cues but on the lookout for social opportunities - which may result in poorer recognition of fearful cues, but heightened identification of affiliation cues in the voice. Examining multiple components of the social information processing stream longitudinally, including an assessment of attentional biases, emotion recognition, and enacted behaviour in social settings, would inform a more complete model of the impact of loneliness in the social sphere.

Future studies should also examine loneliness and ER using multimodal stimuli combining facial and vocal information. Given previous work showing that ambiguity in social situations causes problems for lonely youth (Qualter et al., 2013), presenting stimuli in which the emotions conveyed by the face and voice are incongruent would help determine whether lonely people prioritize information from one modality over the other when social cues are unclear. Future work would also benefit from assessing other emotional skills that can play a role in lonely individuals' responses to socio-emotional information, such as emotion regulation abilities.

\section{Conclusions}

The association between loneliness and the recognition of vocal emotional cues in adolescents was found to be emotion-specific. While loneliness has been linked to an enhanced recognition of negative facial emotions, the current results suggest that a reverse pattern may occur in the recognition of vocal affect. Loneliness was linked to poorer recognition of vocal expressions of fear and heightened identification of friendliness. Findings support theoretical models proposing that loneliness may encourage avoidance of threatening cues but attunement to indices of social affiliation. Being more sensitive to cues of friendliness may promote reconnection opportunities for the lonely person, and represent an adaptive component of the 


\section{LONELINESS \& VOCAL EMOTION RECOGNITION}

social monitoring system. Interventions that encourage youth to use this attentional orientation adaptively may be effective in overcoming loneliness by helping adolescents to focus on social affiliation cues in others' voices.

Conflict of Interest Statement: The authors whose names are listed above certify that they have no affiliations with or involvement in any organization or entity with any financial or non-financial interest in the subject matter or materials discussed in this manuscript. 


\section{LONELINESS \& VOCAL EMOTION RECOGNITION}

\section{References}

Cacioppo, J.T., \& Hawkley, L.C. (2009). Perceived social isolation and cognition. Trends Cogn Sci, $13,447-454$

Chronaki, G., Hadwin, J.A., Garner, M., Maurage, P., \& Sonuga-Barke, E.J.S. (2015). The development of emotion recognition from facial expressions and non-linguistic vocalizations during childhood. British Journal of Developmental Psychology, 33, 218236.

Gardner, W.L., Pickett, C.L., Jefferis, V., \& Knowles, M. (2005). On the outside looking in: loneliness and social monitoring. Pers Soc Psychol Bull, 31, 1549-1560.

Green, M.J., \& Phillips, M.J. (2004). Social threat perception and the evolution of paranoia. Neuroscience \& Biobehavioral Reviews, 28(3), 333-342.

Herba, C., \& Phillips, M. (2004). Development of facial expression recognition from childhood to adolescence: behavioural and neurological perspectives. Journal of Child Psychology and Psychiatry, 45(7), 1185-1198.

Holm, S. (1979). A simple sequentially rejective multiple test procedure. Scandinavian Journal of Statistics, 6(2), 65-70.

Johnstone, T., \& Scherer, K.R. (2000). Vocal communication of emotion. In M. Lewis \& J. Haviland (Eds.), Handbook of emotions (pp. 220-235). New York: Guilford.

Knowles, M.L., Lucas, G.M., Baumeister, R.F., \& Gardner, W.L. (2015). Choking under social pressure: social monitoring among the lonely. Pers Soc Psychol Bull, 41, 805-821.

La Greca, A.M., \& Stone, W.L. (1993). Social anxiety scale for children-revised: Factor structure and concurrent validity. Journal of Clinical Child Psychology, 22, 17-27. 


\section{LONELINESS \& VOCAL EMOTION RECOGNITION}

Lodder, G.M.A., Scholte, R.H.J., Goossens, L., Engels, R.C.M.E., \& Verhagen, M. (2016). Loneliness and the social monitoring system: Emotion recognition and eye gaze in a reallife conversation. British Journal of Psychology, 107, 135-153.

Marcoen, A., Goossens, L., \& Caes, P. (1987). Loneliness in pre-through late adolescence: Exploring the contributions of a multidimensional approach. Journal of Youth and Adolescence, 16, 561-577.

McClure, E.B., \& Nowicki Jr., S. (2001). Associations between social anxiety and nonverbal processing skill in preadolescent boys and girls. Journal of Nonverbal Behavior, 25(1), 318.

Morningstar, M., Dirks, M.A., \& Huang, S. (2017). Vocal Cues Underlying Youth and Adult Portrayals of Socio-emotional Expressions. Journal of Nonverbal Behavior, 41, 155-183.

Morningstar, M., Ly, V.Y., Feldman, L., \& Dirks, M.A. (2018). Mid-Adolescents’ and Adults’ Recognition of Vocal Cues of Emotion and Social Intent: Differences by Expression and Speaker Age. Journal of Nonverbal Behavior, 42, 237-251.

Morningstar, M., Nelson, E.E., \& Dirks, M.A. (2018). Maturation of vocal emotion recognition: Insights from the developmental and neuroimaging literature. Neuroscience \& Biobehavioral Reviews, 90, 221-230.

Nelson, E.E., Leibenluft, E., McClure, E.B., \& Pine, D.S. (2005). The social re-orientation of adolescence: a neuroscience perspective on the process and its relation to psychopathology. Psychological Medicine, 35, 163-174.

Perlman, D., \& Peplau, L.A. (1981). Toward a social psychology of loneliness. Personal Relationships, 3, 31-56. 


\section{LONELINESS \& VOCAL EMOTION RECOGNITION}

Qualter, P., Rotenberg, K., Barrett, L., Henzi, P., Barlow, A., Stylianou, M., \& Harris, R.A. (2013). Investigating hypervigilance for social threat of lonely children. Journal of Abnormal Child Psychology, 41, 325-338.

Qualter, P., Vanhalst, J., Harris, R., Van Roekel, E., Lodder, G., Bangee, M., . . Verhagen, M. (2015). Loneliness across the life span. Perspectives on Psychological Science, 10, 250264.

Rönkä, A.R., Rautio, A., Koiranen, M., Sunnari, V., \& Taanila, A. (2014). Experience of loneliness among adolescent girls and boys: Northern Finland Birth Cohort 1986 study. Journal of youth studies, 17, 183-203.

Spithoven, A.W.M., Bijttebier, P., \& Goossens, L. (2017). It is all in their mind: A review on information processing bias in lonely individuals. Clin Psychol Rev, 58, 97-114.

Vanhalst, J., Gibb, B.E., \& Prinstein, M.J. (2017). Lonely adolescents exhibit heightened sensitivity for facial cues of emotion. Cogn Emot, 31, 377-383.

Wagner, H.L. (1993). On measuring performance in category judgment studies of nonverbal behavior. Journal of Nonverbal Behavior, 17, 3-28.

Yiend, J. (2010). The effects of emotion on attention: A review of attentional processing of emotional information. Cognition and Emotion, 24, 3-47.

Zysberg, L. (2012). Loneliness and emotional intelligence. J Psychol, 146, 37-46. 
LONELINESS \& VOCAL EMOTION RECOGNITION

Table 1

Full factorial general linear model results

\begin{tabular}{lllll}
\hline Effect & $d f$ & $F$ & $\eta^{2}$ & $p$ \\
\hline Loneliness & $(1,115)$ & 0.85 & $<.01$ & .77 \\
Social Anxiety & $(1,115)$ & 0.29 & $<.01$ & .59 \\
Age & $(1,115)$ & 1.21 & .01 & .27 \\
Gender & $(1,115)$ & 4.70 & .04 & .03 \\
Emotion & $(5.47,628.48)$ & 139.84 & .55 & $<.001$ \\
Emotion x Loneliness & $(5.47,628.48)$ & 4.77 & .04 & $<.001$ \\
Emotion x Social Anxiety & $(5.47,628.48)$ & 2.69 & .02 & .02 \\
Emotion x Age & $(5.47,628.48)$ & 5.66 & .05 & $<.001$ \\
Emotion x Gender & $(5.47,628.48)$ & 1.04 & .01 & .39 \\
\hline
\end{tabular}

Note. $\eta^{2}=$ partial eta squared. Loneliness, Social Anxiety, and Age were mean-centered continuous predictors. 

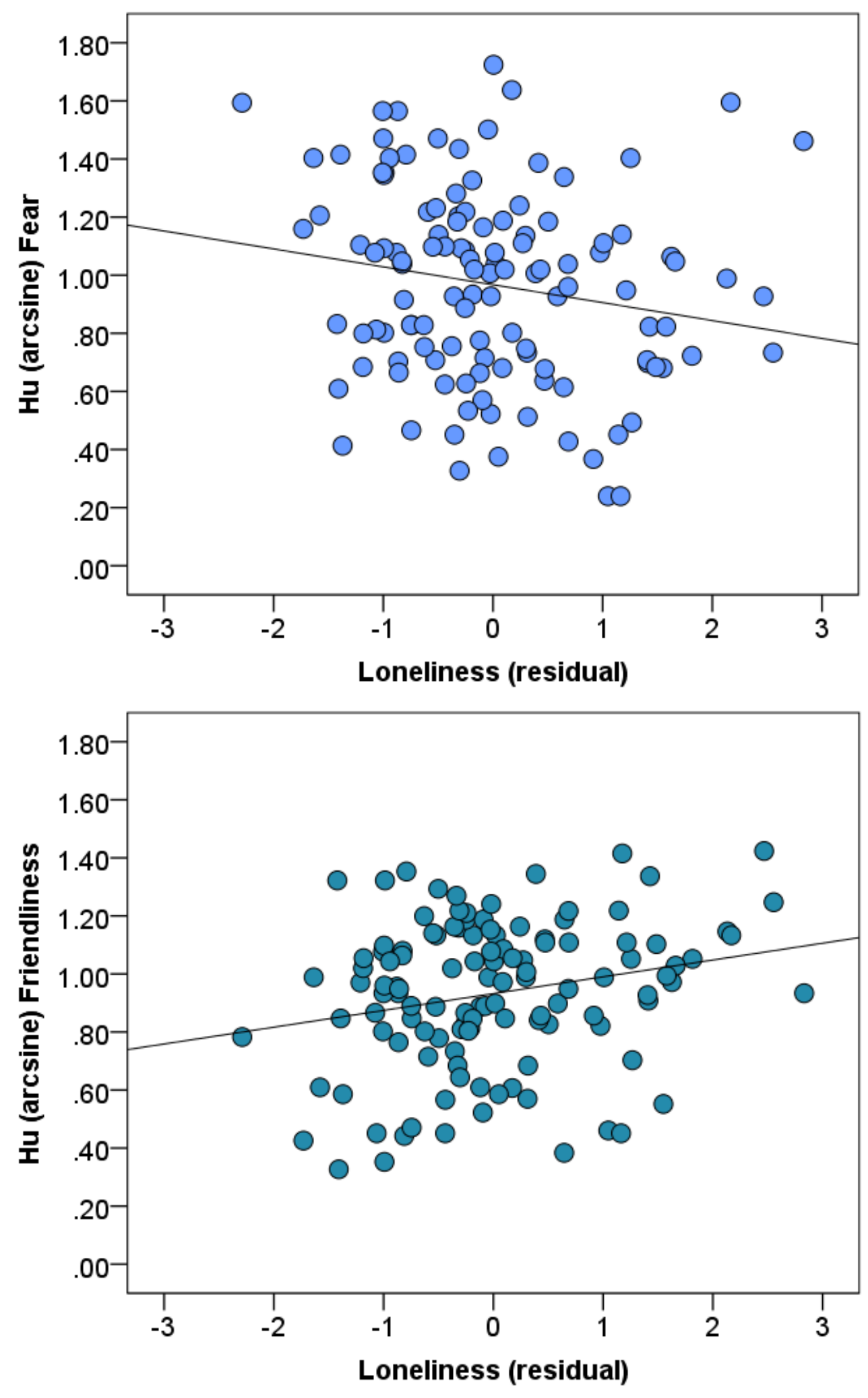


\section{LONELINESS \& VOCAL EMOTION RECOGNITION}

\section{Figure caption}

Fig. 1 Scatterplot representing the relationship between the standardized residual for meancentered Loneliness scores (residualized on mean-centered Age, Gender, and mean-centered Social Anxiety scores) and recognition accuracy $\left(\mathrm{H}_{\mathrm{u}}\right.$; unbiased hit rate, arcsine transformed) for fear (top) and friendliness (bottom). 